\title{
A Rare Case of Primary Cutaneous Apocrine Carcinoma
}

\author{
Persechino $\mathrm{F}^{1}$, Giordano $\mathrm{D}^{2}$, Gagliostro $\mathrm{N}^{2}$, Capalbo $\mathrm{AC}^{2}$, Balabanos $^{2}$, Severino Persechino ${ }^{2 *}$
}

\author{
${ }^{1}$ Department of Experimental Medicine, Sapienza University \\ of Rome, Rome, Italy.
}

${ }^{2} N E S M O S$ Department, Dermatology Unit, Sant'Andrea Hospital Faculty of Medicine and Psychology University of Rome "Sapienza", Italy.

\section{${ }^{*}$ Corresponding author}

Severino Persechino, NESMOS Department, Dermatology Unit, Sant’Andrea Hospital Faculty of Medicine and Psychology University of Rome "Sapienza", Italy.

Submitted: 19 Aug 2020; Accepted: 15 Sep 2020; Published: 22 Oct 2020

\begin{abstract}
Primary cutaneous apocrine carcinoma is a rare malignant tumor that originates from apocrine sweat glands. Apocrine sweat gland tumor is infrequent: the first case was published by Horn in 1944 and less than 50 cases are described in literature worldwide. For this reason, there are no clear guidelines for the management and treatment of this uncommon cancer. We report a clinical case of primary cutaneous apocrine carcinoma in a 86 year-old female patient.
\end{abstract}

\section{Introduction}

Primary cutaneous apocrine carcinoma is a rare malignant tumor that originates from apocrine sweat glands. Generally, apocrine sweat glands are located to anogenital area and axilla, but they are found as modified glands also in mammary areola, in eyelid (Moll's glands) and ear canals (ceruminous glands). Apocrine carcinoma of skin is usually observed in adults, without differences between sex and ethnic groups and its risk factors are unknown. It usually presents as a slow-growing solitary nodule in the axilla region, but some cases of rapidly progressive and aggressive tumor are reported [1-3]. It can be locally advanced with metastasis to local lymph nodes or it can be metastatic. The treatment of choice is surgical excision with or without lymph node dissection, followed by chemotherapy and radiation therapy, if necessary. We present a case of an 86-year female patient with primary cutaneous apocrine carcinoma located to right axilla and mammary area with lymph nodes and chest metastasis and neoplastic obliteration of subclavian vein.

\section{Case Report}

We present the clinical case of an 86-year-old woman, hospitalized in our Department for an extended cutaneous lesion of the right axillary and mammary region. As referred by the patient, the cutaneous lesion was present for four months.

At the clinical examination, we observed multiple infiltrated crusted-scaly lesions of variable shape and size, localized on the right external mammary region. The cutaneous perilesional region, involving breast, axillary cord and partially the right upper limb, appeared hot, erythematous, edematous and xerotic. Right axil- lary and latero-cervical lymph nodes were palpable, painful and low-lying compared to the superficial and deep planes.

The patient reported a rapid growth of the lesion in the last four months, associated with a progressive development of edema, regional lymphadenopathy and local pain. Firstly, ultrasonography and mammography exams were performed in order to exclude a cutaneous metastatic breast tumor. Both exams resulted negative. After that we did a chest X-ray, which was negative for lung tumors. Moreover, we performed an upper extremities Doppler ultrasound to exclude thrombotic factors as a cause of the clinical condition. The exam, once again, did not show vascular alterations. Laboratory tests were normal, except for a slight increase in fibrinogen and gamma proteins.

Since all the exams resulted negative, we performed an incisional biopsy of the cutaneous lesion. The histological examination revealed: "Malignant tumor with epithelial phenotype and acantholytic aspects (panCK-positive and negative melanocytic markers) with aspects of intraepithelial pagetoid dissemination, suggesting carcinoma originated from apocrine adnexal structures. The lesion infiltrates the dermis".

Considering the histological exam result, we performed a wholebody computed tomography for tumor staging. The exam highlighted solid tissue localized on the right axillary region, infiltrating large and small round muscles, subscapularis and infraspinatus muscles, pectoral muscle, large dorsal muscle, scalene muscle and sternocleiodomastoid muscle. In the latero-cervical region, this solid tissue wrapped the right subclavian vein, causing compres- 
sion and obliteration. The exam showed also a solid nodule localized on the right axillary cavity and a nodule at the inferolateral quadrant of the right breast, with associated thickening of cutaneous layers. Moreover, the exam revealed multiple millimetric micronodules localized on the lung basal segments, bilaterally, and a hypodense lesion situated on the left occipital region.

Since the neoplastic nature of the disease, the extended and infiltrative characteristics of the tumor, metastasis and the bad general conditions of the patient, a palliative radiotherapy treatment of lesions and lymphedema (4 Gy x 5) was chosen.

\section{Discussion}

Primary cutaneous apocrine carcinoma is a rare and highly aggressive tumor that arises from apocrine sweat glands. Apocrine sweat gland tumor is really infrequent. Actually, the first case was published by Horn in 1944 and less than 50 cases are described in Literature worldwide [1-6]. For this reason, there are no clear guidelines for the management and treatment of this uncommon cancer. Generally, apocrine sweat gland carcinoma affects adults, without predilection for sex or race. Its pathogenesis is unknown and certain risk factors are not reported in Literature [7].

It is usually located to axilla, because of high density of sweat glands in this area, but some cases of apocrine carcinoma on scalp, nipple, anogenital region and chest are reported in Literature. It usually presents as a slow-growing solitary nodule or plaque and ulceration and bleeding are common associated findings. Metastasis to lymph nodes and parenchymal organs can also occur. Primary axillary apocrine adenocarcinoma can be confused with other more frequent clinical conditions, such as metastatic breast cancer and extra-mammary Paget disease. For this reason, a cutaneous biopsy with a histological exam is always necessary.

Concerning therapy, the treatment of apocrine adenocarcinoma is based on surgical excision with a 1-2 cm free margin of normal tissue along with or without regional lymph node dissection.

Chemotherapy and radiation therapy can be useful in metastatic disease, even if patients with metastatic progression have a very poor prognosis. High incidence of local recurrence is reported in Literature and prophylactic nodal dissection does not reduce this phenomenon [8].

The prognostic factors for apocrine adenocarcinoma are relatively poor and include staging, histological type, lymph node involvement, distant metastasis, age and response to treatment.

\section{Conclusion}

We describe this case to underline the rarity of primary cutaneous apocrine carcinoma: less than 50 cases are described in literature worldwide. For this reason, there are no clear guidelines for the management and treatment of this uncommon cancer. Dermatologists should be aware of the existence of apocrine carcinoma and they should suspect this uncommon entity in presence of a cutaneous nodule located in the axillary region. Multidisciplinary approach is essential for management and treatment of this patients and regular follow up should be encouraged.
Informed consent: Informed consent from the patient has been obtained to publish the photographs shown in Figure 1, 2 and 3.

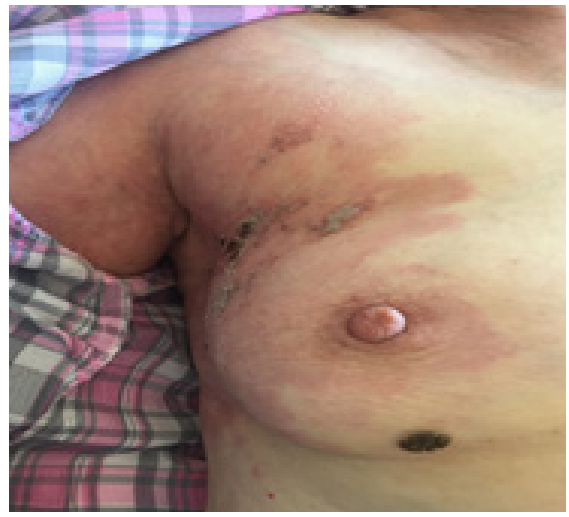

Figure 1: Multiple infiltrated crusted-scaly lesions of variable shape and size, localized on the right external mammary region. The perilesional region appeared hot, erythematous, edematous and xerotic.

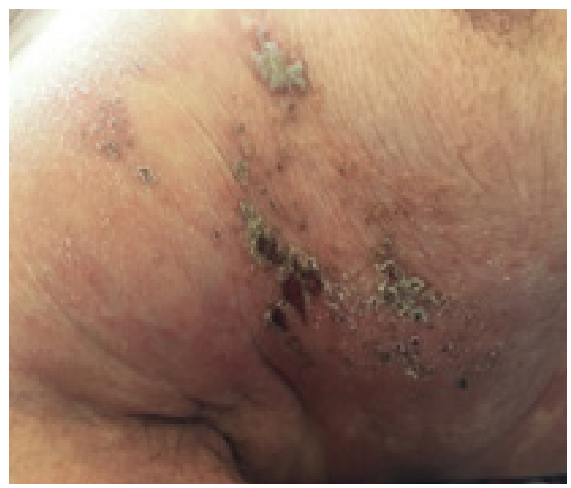

Figure 2: Multiple infiltrated crusted-scaly lesions of variable shape and size, localized on the right external mammary region. The perilesional region appeared hot, erythematous, edematous and xerotic.

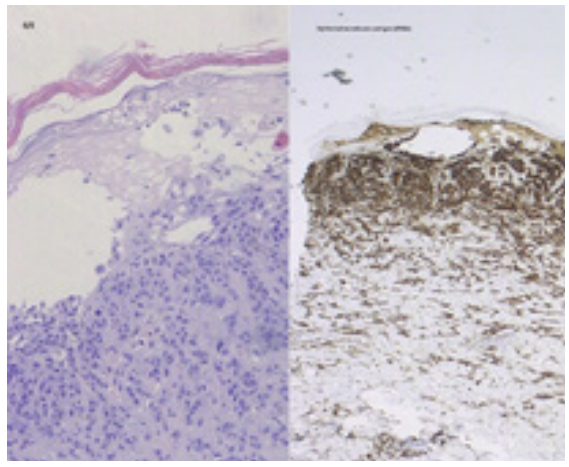

Figure 3: Malignant tumor with epithelial phenotype and acantholytic aspects (panCK-positive and negative melanocytic markers) with aspects of intraepithelial pagetoid dissemination, suggesting carcinoma originated from apocrine adnexal structures. The lesion infiltrates the dermis. 


\section{References}

1. Karaca S, Kulac M, Sahin O, Esme H, Solak O, et al. (2007) A rare cutaneous tumor of the axilla: Apocrine adenocarcinoma. Indian Journal of dermatology 52: 50-52.

2. Seong MK, Kim EK, Han K, Seol H, Kim HA, et al. (2015) Primary apocrine sweat gland carcinomas of the axilla: a report of two cases and a review of the literature. World Journal of Surgical Oncology 13: 59.

3. Zahid R, Soofi ME, Elmalik H, Junejo K (2016) Primary apocrine carcinoma of the axilla in a male patient: a case report. Clin Case Rep 4: 344-347.

4. Horn RC (1944) Malignant papillary cystadenoma of sweat glands with metastases to the regional lymph nodes. Surgery 16: 348-355.

5. Vasilakaki T, Skafida E, Moustou E, Grammatoglou X, Arkoumani E, et al. (2011) Primary Cutaneous Apocrine Carcinoma of Sweat Glands: A Rare Case Report. Case Rep Oncol 4: $597-601$.

6. Seung-Hee Loh, Yu-Jin Oh, Bark-Lynn Lew, Woo-Young Sim (2016) Primary Cutaneous Apocrine Carcinoma. Ann Dermatol 28: 669-670.

7. Paredes Gatti A, Tonello L, Pfaffenzeller W, OliveiraSavóia F, Goergen DI, et al. (2017) Apocrine sweat gland adenocarcinoma: A rare case report and review. Int Jour Surgery Case Reports 36: 78-81.

8. Schweitzer J, Vermeesch J, Zaleski T, Megan Iacco, Kent Krach, et al. (2016)Primary cutaneous apocrine carcinoma: 2 cases and review of the pertinent histologic findings. JAAD Case Reports 2: 411-414.

Copyright: (C2020 Severino Persechino., This is an open-access article distributed under the terms of the Creative Commons Attribution License, which permits unrestricted use, distribution, and reproduction in any medium, provided the original author and source are credited. 